\title{
Lyapunov based stability analysis for metro lines
}

\author{
A. Berbey ${ }^{2,3}$, R. Galán ${ }^{2}$, P. San Segundo ${ }^{2} \&$ J. Sanz-Bobi ${ }^{1}$ \\ ${ }^{1}$ Research Centre in Railway Technologies (CITEF), \\ Universidad Politécnica de Madrid, Spain \\ ${ }^{2}$ The Intelligent Control Group (ICG), \\ Universidad Politécnica de Madrid, Spain \\ ${ }^{3}$ Technological University of Panama, Panama
}

\begin{abstract}
In this work a direct method to measure the stability of metro system lines with respect to a previously constructed time schedule is presented. For this purpose we first model saturation effects using a real time discrete space state representation and then apply a Lyapunov-based stability analysis considering time delays of trains as disturbances. As a result we have been able to define a new set of indexes that relate time delays with the validity of the actual time schedule when falling inside a particular 'stability area'. Results obtained in a simulated environment show that the new stability indexes are able to evaluate quantitatively and qualitatively the effects of saturation in metro lines as well as predict the need for rescheduling
\end{abstract}

Keywords: metro system, stability, planning, genetic algorithm, artificial intelligence.

\section{Introduction}

The dynamics of metro line systems have been deeply studied by several researchers [1-5,7-10]. Most of these dynamical models are based on the Sasama and Ohkawa [9] linear model.

It is well known that such kind of linear models, usually yield simple formulation, implementation and simulation. As a result, some dynamic traffic linear controllers $[1-3,7]$ and real time simulators $[3,4]$ have been proposed. 
Some researchers [4,5] state the importance of nonlinear behaviour of metro lines. Therefore, a suitable nonlinear stability analysis must be done in order to design appropriate dynamic traffic controllers.

The Intelligent Control Group (ICG) in collaboration with the Research Centre in Railway Technologies (CITEF) of the Technical University of Madrid, has been working in the development of a railway traffic planning system for metro lines in the last years.

The developed system carries out the following functions:

1) Obtaining any necessary input data by means of a suitable user interface (objective frequencies of sets of trains, number of trains available and the departure stations of these trains, topology of the network, etc.)

2) A plan is generated using the minimum waiting times and the planned running times between stations.

3) If the frequency of trains is greater than the demanded frequency it builds a new plan with possibly the insertion of additional trains.

4) If the frequency of trains is below the demanded frequency it recovers the plan with fewer trains.

5) If the frequency is acceptable, the plan is validated with a simulator that contains the behaviour model of different sets of trains, the railway network and the energy analysis according to any available power plants and the power consumption in each trajectory.

6) If there are discrepancies between calculated times by the simulator and those established by the selected plan, it updates the necessary times for each kind of train in each travel with the ones obtained through simulation and again reschedules (it is also possible to employ times taken from real data for rescheduling).

7) Once a plan has been obtained iteratively by a combination of the previous steps, it is then possible to analyze the consequences of delays in one or several trains arriving at different stations. The delay of a train in leaving a station could be due to: an increase of the number of passengers, technical difficulties, security in the starting of the train, or any other delay on the travel. Our new approach enables us to predict the number of affected trains and the time it will take to re-establish the normal frequencies of the affected trains in the system. Furthermore our approach allows us to define for any plan a valid working zone. As a result, once previously mentioned anomalies take the system outside the plan's valid working area it is no longer valid.

8) A specific module deals with rescheduling in the presence of serious anomalies like train break downs, blockade of a line etc.

This article analyses in detail the stability analysis module, which not only considers control saturation effects, but also the system stability in presence of disturbances. The analysis is based on a new set of stability indexes specifically designed for metro lines which is well known to be a hard real time planning environment. 


\section{Metro line modelling}

\subsection{Traffic dynamical model}

The metro system can be modelled by a system of equations in discreet time [9],

$$
t_{k+1}^{i}=t_{k}^{i}+r_{k}^{i}+s_{k+1}^{i}
$$

where $t_{k}^{i}$ it is the departure time of train i of station $k, r_{k}^{i}$ is the running time of train $i$ of station $k$ to station $k+1, s_{\mathrm{k}}^{i}$ it is the stopping time of train $i$ of station $k$.

It has been demonstrated that metro lines are unstable in presence of disturbances if no control action is applied for correcting the itinerary of the trains $[1,2]$. Such kind of control action could be performed by manipulating stop times or in-between station running times. In other words, both actions are functionally equal [5]. If the running time is manipulated, it is defined by,

$$
r_{k}^{i}=R_{k}+u_{R k}^{i}
$$

where $R_{k}$ it is the nominal running time which takes a train to reach station $k+1$ coming from station $\mathrm{k}$ and $u_{R k}^{i}$ is the control action on the train $i$ in the route between the stations $k$ and $k+1$.

\subsection{State space model}

Scheduling in ideal conditions (without disturbances [1,2]) is defined by equations $u_{R k}^{i}=0$ and $t_{k+1}^{i}=\mathrm{T}_{\mathrm{k}+1}^{\mathrm{i}}$, where $\mathrm{T}_{\mathrm{k}+1}^{\mathrm{i}}$ is a previously established adequate timing. Disturbances in the model are captured by parameter $x_{k}^{i}=\mathrm{T}_{\mathrm{k}}^{\mathrm{i}}-t_{\mathrm{k}}^{\mathrm{i}}$ which is the difference between the scheduled timing per station and the real time.

If we consider an ideal planning situation as an equilibrium point we can reformulate the problem as a discreet state space representation as follows:

$$
\begin{aligned}
& X_{j+1}=A X_{j}+B U_{j}+W_{j} \\
& Y=X_{j}
\end{aligned}
$$

where $X_{j}=\left[\begin{array}{lll}x_{1}^{j-1} & \cdots & x_{K}^{j-K}\end{array}\right]^{T} ; \quad U_{j}=\left[\begin{array}{lll}u_{0}^{j} & \cdots & u_{K-1}^{j-K+1}\end{array}\right]^{T} ; \quad W_{j}=\left[\begin{array}{lll}u_{0}^{j} & \cdots & u_{K-1}^{j-K+1}\end{array}\right]^{T}$

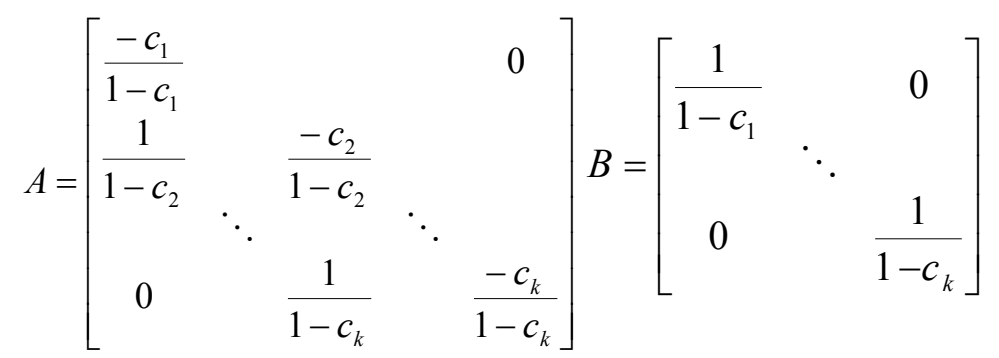

The terms $w_{k+1}^{i}$ correspond to the disturbances, inherent to both models and $c_{k+1}$ is the delay rate capturing the effect of time intervals between departures of two successive trains. 
Such dynamical system can be stabilized with the following suboptimal ideal linear controller,

$$
U_{j}=\phi_{j} X_{j}
$$

where $\phi_{j}=\left[\begin{array}{cccccc}f & 0 & 0 & 0 & 0 & 0 \\ g & f & 0 & 0 & 0 & 0 \\ 0 & g & f & 0 & 0 & 0 \\ 0 & 0 & g & f & 0 & 0 \\ \vdots & \vdots & \vdots & \vdots & \ddots & \vdots \\ 0 & 0 & 0 & 0 & g & f\end{array}\right]$

The $f$ and $g$ parameters could be estimated employing the following formulation,

$$
\begin{aligned}
& f=\frac{q+p c_{c}}{\left(1-c_{c}\right)^{2}+p+q} \\
& g=-\frac{p+q}{\left(1-c_{c}\right)^{2}+p_{c}+q_{c}}
\end{aligned}
$$

where $0 \leq p<\infty ; 0 \leq q<\infty$ and $c_{c}$ is the maximum value of $c_{k}$.

Nevertheless, it is important to stress that the control actions $U_{j}$ are translated in automatic corrections in the stopping times and/or speeds of the trains. These actions usually are limited by inherent restrictions of stoppage times in the stations and the speeds of the trains on the track. It is possible to model these saturated control actions as follows:

$$
\begin{gathered}
U_{j}=\phi\left(X_{j}\right) X_{j} \\
\text { where } \phi\left(X_{j}\right)=\left[\begin{array}{cccccc}
f_{1}^{\prime} & 0 & 0 & 0 & 0 & 0 \\
g_{2}^{\prime} & f^{\prime}{ }_{2} & 0 & 0 & 0 & 0 \\
0 & g^{\prime} & f^{\prime}{ }_{3} & 0 & 0 & 0 \\
0 & 0 & g^{\prime} & f^{\prime}{ }_{4} & 0 & 0 \\
\vdots & \vdots & \vdots & \vdots & \ddots & \vdots \\
0 & 0 & 0 & 0 & g^{\prime}{ }_{k}^{\prime} & f^{\prime}
\end{array}\right] \\
f_{k+1}^{\prime}= \\
g_{k+1}^{\prime}=\left\{\begin{array}{ccc}
f & \text { for }-K_{k} \leq g x_{k}^{i}+f x_{k+1}^{i-1} \\
\frac{K_{k} f}{\left|g x_{k}^{i}+f x_{k+1}^{i-1}\right|} & \text { for } g x_{k}^{i}+f x_{k+1}^{i-1}<-K_{k} \\
\frac{g}{\left|g x_{k}^{i}+f x_{k+1}^{i-1}\right|} & \text { for } g x_{k}^{i}+f x_{k+1}^{i-1}<-K_{k}
\end{array}\right.
\end{gathered}
$$




\section{Nonlinear stability analysis}

The previous analysis allows us to determinate a Lyapunov positive defined function

$$
V\left(X_{j}\right)=X_{j}^{T} X_{j}
$$

This dynamical system will be stable if

$$
\Delta V=V\left(X_{j+1}\right)-V\left(X_{j}\right)<0
$$

We have been able to demonstrate that (8) is satisfied for any

$$
1-r^{2}\left(-c_{c}+f_{k+1}^{\prime}\right)^{2}-r^{2}\left(1+g_{k+1}^{\prime}\right)^{2}>\left|r^{2}\left(-c_{c}+f_{k}^{\prime}\right)\left(1+g_{k+1}^{\prime}\right)\right|+\left|r^{2}\left(-c_{c}+f_{k+1}^{\prime}\right)\left(1+g_{k+2}^{\prime}\right)\right|
$$

where $r^{2}=\max \left(1-c_{k+1}\right)^{-2}=\left(1-c_{c}\right)^{-2}$

This result is not included here because of lack of space, but will be the subject of a future paper.

As a result, three important zones of stability in the system appear:

a) First zone: it is a linear zone with guaranteed stability and it is defined by, $g_{k+1} x_{k}^{i}+f_{k+1} x_{k+1}^{i-1} \geq-K_{k}$

b) Second zone: it is a nonlinear zone with guaranteed stability and it is defined by, $1-r^{2}\left(-c_{k+1}+f_{k+1}^{\prime}\right)^{2}-r^{2}\left(1+g_{k+1}^{\prime}\right)^{2}>r^{2}\left(-c_{k}+f_{k}^{\prime}\right)\left(1+g_{k+1}^{\prime}\right)|+| r^{2}\left(-c_{k+1}+f_{k+1}^{\prime}\right)\left(1+g_{k+2}^{\prime}\right) \mid$ where $g x_{k}^{i}+f x_{k+1}^{i-1}<-K_{k}$

c) Third zone: it is a nonlinear zone where stability is not guaranteed and is formally defined by

$$
1-r^{2}\left(-c_{k+1}+f_{k+1}^{\prime}\right)^{2}-r^{2}\left(1+g_{k+1}^{\prime}\right)^{2} \leq r^{2}\left(-c_{k}+f_{k}^{\prime}\right)\left(1+g_{k+1}^{\prime}\right)|+| r^{2}\left(-c_{k+1}+f_{k+1}^{\prime}\right)\left(1+g_{k+2}^{\prime}\right) \mid
$$

where $g x_{k}^{i}+f x_{k+1}^{i-1}<-K_{k}$

The intuition behind the previous stability analysis states is that saturated controllers are able to stabilize metro lines in the first and second zone. However, the linear controller stability is uncertain in the third zone. These results are in accordance with experimental data obtained from real systems [4].

\section{A new stability index}

In the authors' opinion, it would be very useful to know in advance the "fragility" of a linear base controller used for traffic regulation. Our previous formulation allows us to define a new set of stability indexes based on the distance from the origin (zero error) to the borders between the second (nonlinear stable) and third (non-guaranteed stability) zone (see figure 1):

$$
v_{k+1}^{i}=\frac{\left|r^{2}\left(-c_{k}+f_{k+1}^{\prime}\right)\left(1+g_{k+1}^{\prime}\right)\right|+\left|r^{2}\left(-c_{k+1}+f_{k+1}^{\prime}\right)\left(1+g_{k+2}^{\prime}\right)\right|}{1-r^{2}\left(-c_{k+1}+f_{k+1}^{\prime}\right)^{2}-r^{2}\left(1+g_{k+1}^{\prime}\right)^{2}}
$$




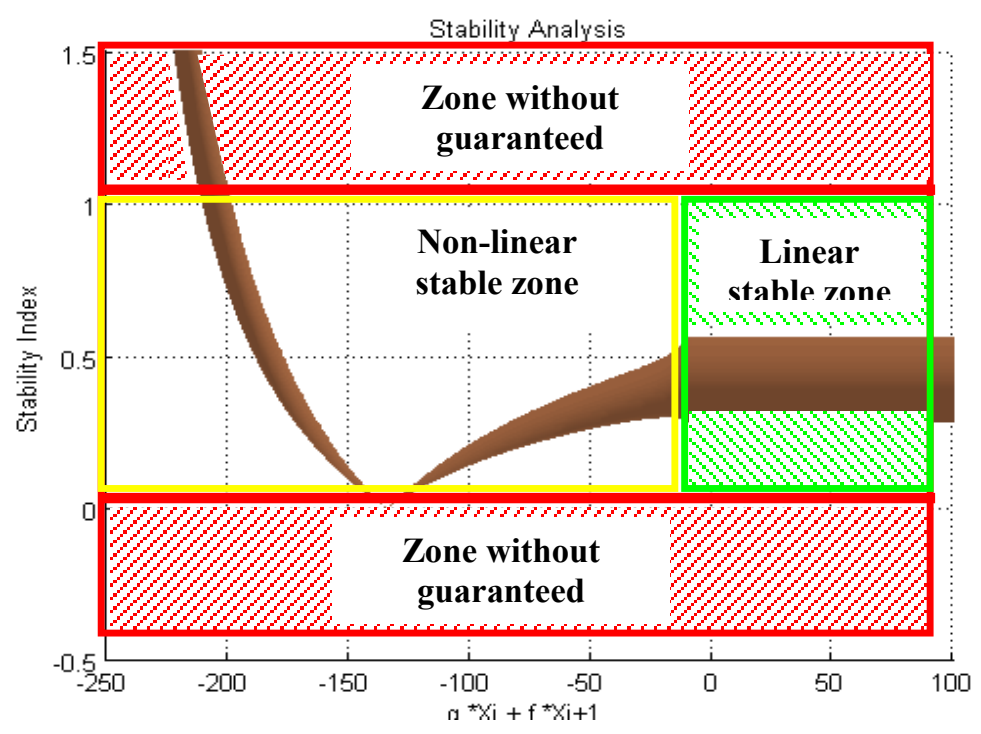

Figure 1: Stability zones.

The dynamical system will have guaranteed stability if each of the indexes are in the interval:

$$
0 \leq v_{k+1}^{i}<1
$$

In case that any $v_{k+1}^{i}$ is negative or equal or greater than one the system stability will not be guaranteed and the metro line could be unstable and need rescheduling.

\section{Experiments}

To validate our approach we have carried out a number tests in a simulated environment using a tool was developed in MATLAB ${ }^{\circledR}$ to evaluate the stability in different scenarios.

Starting from platform 6 and the fiftieth train, we introduced four delay time disturbances of 50,200, 250 and 2000 seconds respectively using suboptimal values $p=1, q=1, c_{c}=0.026$, and $K_{k}=8.4$ to compute the parameters of the discreet space state model.

In the first case, the metro line is disturbed with 50 seconds delay (figure 2). The system shows light deviations in three trains and stability indexes $v_{k+1}^{i}$ reaching values between 0.3 and 0.50 . This means that the metro line system is inside the stability zone with a possible increment of 0.50 from the upper nonguaranteed stability threshold (zone 2) and 0.30 from the lower non-guaranteed stability threshold.

A 200 seconds delay produces values for the stability indexes between 0.1 and 0.60 (figure 3). A critical stability point is reached for a 250 seconds disturbance (figure 4), as the system reaches the border between the second and third zones. Finally a 2000 seconds disturbance on the metro line makes the 
saturation effects so important that they are able to destabilize the system (figure 5). It is important to highlight that the proposed index is able to predict in advance (around 250 seconds after disturbance has started) the possibility of the system becoming unstable and the need to reschedule.

The summarized results are presented in table 1 . In case of a 2000 second delay, the recovering time is about $40 \times 10^{4}$ seconds because there is a big disturbance that affects an average of 38 trains approx. It is for this reason that index $v_{k}^{i}$ falls outside the second zone which guarantees stability.

Table 1: $\quad$ Experimental results.

\begin{tabular}{|c|c|c|c|c|c|}
\hline \multirow[t]{2}{*}{ Disturbances } & \multirow{2}{*}{$\begin{array}{l}\text { Trains } \\
\text { affected }\end{array}$} & \multicolumn{2}{|c|}{$v_{k}^{i}$} & \multirow[t]{2}{*}{ Zone } & \multirow{2}{*}{$\begin{array}{c}\text { Recovering } \\
\text { time(s) }\end{array}$} \\
\hline & & Min & $\max$ & & \\
\hline 50 & 3 & 0.30 & 0.50 & Stable zone & 400 \\
\hline 100 & 4 & 0.22 & 0.52 & Stable zone & 600 \\
\hline 200 & 5 & 0.10 & 0.60 & Stable zone & 800 \\
\hline 250 & 6 & 0.00 & 0.57 & $\begin{array}{c}\text { Stable zone } \\
\text { (critical point) }\end{array}$ & 1000 \\
\hline 1000 & 12 & -60 & 30 & $\begin{array}{l}\text { Stability not } \\
\text { guaranteed }\end{array}$ & 2000 \\
\hline 2000 & 38 & -8 & 80 & $\begin{array}{l}\text { Stability not } \\
\text { guaranteed }\end{array}$ & $40 \times 10^{4}$ \\
\hline
\end{tabular}
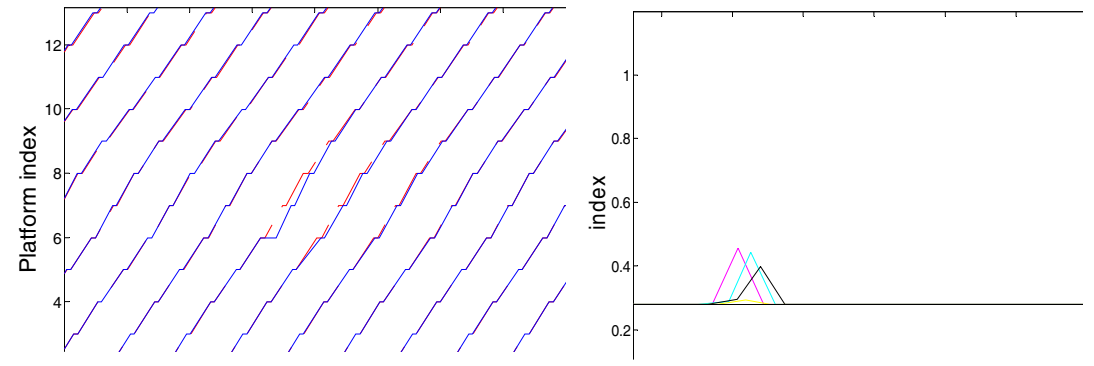

Figure 2: $\quad$ Timetable with disturbance around 50 seconds.
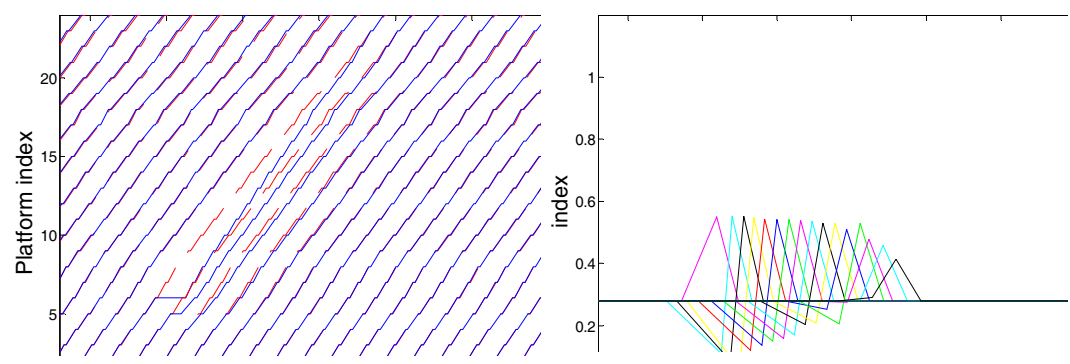

Figure 3: $\quad$ Timetable with a delay disturbance around 200 seconds. 

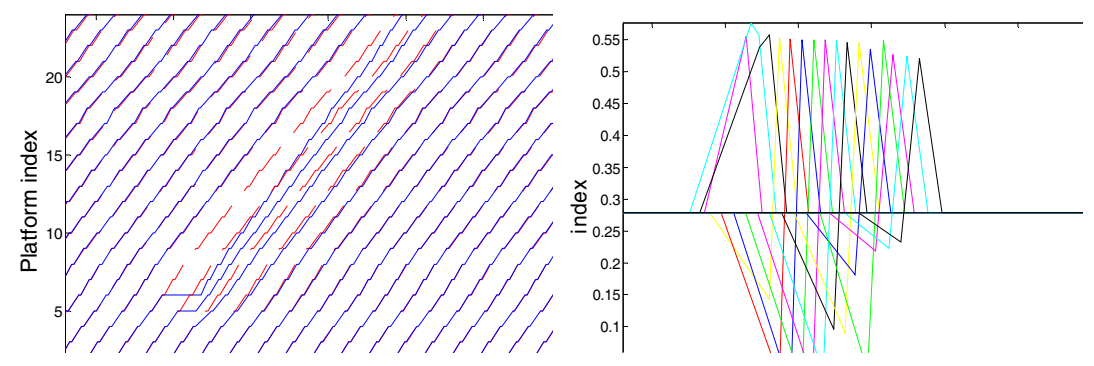

Figure 4: Timetable with a delay disturbance around 250 seconds: critical point.
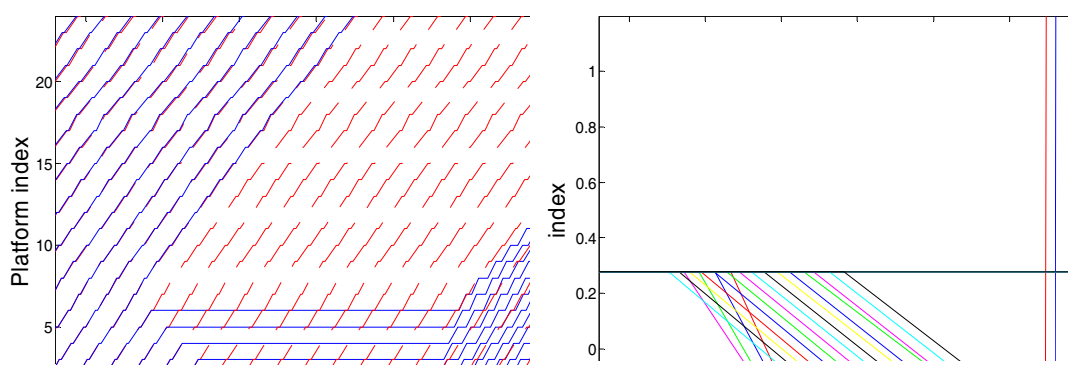

Figure 5: Timetable with a delay disturbance around 2000 seconds: system unstable.

Also is clear that increasing $K_{\mathrm{k}}$ the saturation effects diminishes. Although, this action strengths the dynamical system, there is a trade off between $K_{\mathrm{k}}$ margins and the metro time journey. Too high $K_{\mathrm{k}}$ margins could produce dissatisfaction in metro users.

\section{Conclusions}

It is a fact that railway traffic in general and metro lines in particular show an inherent time delay disturbance from a previously planned time-schedule over time. We have presented a new Lyapunov-based stability analysis over a discreet space state representation of traffic in metro lines. This new approach has allowed us to define a new stability index and three zones of stability: a linear zone, a nonlinear zone with guaranteed stability and a nonlinear zone where stability is not guaranteed. Furthermore, by computing the stability index in real time it would be theoretically possible to predict well in advance the need for global rescheduling.

To validate our approach we have carried out a number of tests with varying time delays in a simulated environment. Future tests on real data are needed for a final assessment, but results obtained are very encouraging. 


\section{Acknowledgement}

A. Berbey is a fellowship holder for doctoral studies of the program of SENACYT-IFARHU 2005-2010 of the government of the Republic of Panama.

\section{References}

[1] V. Van Breusegem, G. Campion and G. Bastin. "Traffic Modeling and State Feedback Control for Metro Lines.”_ IEEE Transactions on automatic control, Vol. 36, No.7, July 1991.

[2] G. Campion, V. Van Breusegem, P. Pinson and G. Bastin. "Traffic Regulation of an underground railway transportation system by state feedback." Optimal control Applications \& Methods, Vol. 6, 385-402. 1985.

[3] F. de Cuadra, A. Fernández and J.C. Granados. "Train simulation and headway calculations an approach based on parameterized continuous curves." Instituto de Investigación Tecnológica (IIT-UPCO) 1994. DIMETRONICS S.A. Madrid, España. Computers in Railways IV-Volume 1: Railway Design and Management. Computational Mechanics Publications.

[4] Fernández, A., de Cuadra, F. y Montes, F. "Traffic regulation and simulation- a predictive adaptive control system." Instituto de Investigación Tecnológica (IIT-UPCO) 1994. DIMETRONICS S.A. Madrid, España. Computers in Railways $I V$-Volume 1: Railway Design and Management. Computational Mechanics Publications.

[5] Araya, S and Sone, S. "Traffic Dynamics of Automated Transit Systems with Pre-established Schedules". IEEE Transactions on Systems, Man and Cybernetics, Vol. SMC-14, No.4, July/August 1984.

[6] Medio, Alfredo(2001): "Non-Linear Dynamics." PortChester, NY, USA: Cambridge University Press, 2001, p 77.

[7] Cury, J.E. Gomide, F.A.C and Mendes, M.J. "A methodology for Generation of Optimal Schedules for an Underground Railway Systems" IEEE Transactions on automatic control, Vol. Ac-25, No.2, April 1980.

[8] Rice, P. " Urban transport systems capacity with special references to London Transport underground railways" Conf. on Control Aspects of New Forms of Guided Land Transport, London, August 1974.

[9] Sasama, H. and Ohkawa, Y. "Floating traffic control for public transportation systems" Proc. $4^{\text {th }}$ IFAC Conf. on Contr. and Transport Syst., Baden-Baden, April 1983.

[10] Assis, W.O; Milani, B.E.A. "Generation of optimal schedules for metro lines using model predictive control" Automatica 40(2004) 13971404.2004. 